\title{
MOOC-BASED CURRICULUM MODEL VALIDATION IN HIGHER EDUCATION IN IRAN
}

\author{
Dr. Esmaeil JAFARI \\ Department of Technological Learning \\ Shahid Beheshti University \\ Tehran, Iran \\ Dr. Kourosh Fathi VAJARGAH \\ Faculty of Education and Psychology \\ Shahid Beheshti University \\ Tehran, Iran \\ Dr. Mahboobeh AREFI \\ Faculty of Education and Psychology \\ Shahid Beheshti University \\ Tehran, Iran \\ Dr. Morteza REZAEIZADEH \\ Faculty of Education and Psychology \\ Shahid Beheshti University \\ Tehran, Iran
}

\section{ABSTRACT}

The aim of this study is to validate MOOC-based curriculum in higher education in Iran. The research method is mixed and of exploratory type. Method of grounded theory is used in the qualitative section and the Validation Test Pattern among the faculties and PhD students of Tehran's Universities is considered in the quantitative part. The statistical population of the qualitative section include 14 experts in educational technology and the quantitative section include 214 members of the faculties and PhD students of Tehran's Universities who were chosen by Stratified random sampling. To collect data, we used semi-structured interview in the qualitative section and scholar's questionnaire in the quantitative part. Results show that there are $\mathbf{2 8}$ general issues in paradigmatic model is obtained which included Terms of cause, Central phenomenon, Underlying conditions, Confounding conditions, Strategies and Implications that reflected effective factor in MOOC-based Curricula in higher education.

Keywords: Higher education, MOOC-based curricula, data-based approach, validation.

\section{INTRODUCTION}

Although from more than two thousand years till now, fields of teaching and learning in comparison with other categories has little changes, assisting information technology it has rapid developments (Jerry, 2000). In recent years, virtual training was mentioned as an important applications of new technologies of information and communication in the world and extensive activities was started in this regard. According to the sweeping changes which taking place around, implementation of virtual systems in order to provide new services and technologies in the field of teaching and learning has been proposed as a fundamental requirement (Ong and et al, 2004). 
Virtual training makes new paradigm and provides possibility of learning in any aspect, for everyone, in any time and any situation (Khan, 2004). Between different forms of virtual training in recent years, extensive and free online courses (MOOC) has substantial expansion (Siemens \& Kathleen, 2012). These courses, especially higher education for adults, caused significant changes. MOOC causes the fundamental positive changes in the curriculum of higher education and in spite of their benefits in providing learning opportunities, it could make challenges and threats for higher education especially in developing countries (McAuley and et al, 2010). The extensive online courses (MOOC) could be a threat to the independence and autonomy of traditional higher education by its flexibility and accessibility (Beránek \& Remes, 2012). These challenges and opportunities imply necessity and validation for MOOC-based curricula in higher education and affect all components of this type of curriculum. With good management and good infrastructure these challenges in order to optimal use of the opportunities, we could take full advantage of MOOC capacity in curricula and increase the effectiveness of higher education.

Identifying these threats and adopting appropriate strategies for managing them would help custodians the authorities and program planners of higher education to achieve MOOC's goals in education system. Thus, it is important the changes that caused by this phenomenon on MOOC-based curricula in higher education, considered in a deep study, so that we could investigate its implications and influences on the key elements of this type of curriculum in the country's educational system which based on the results of this study. Policies adopted by the higher education strongly affected on curriculum as a subsystem of higher education and have influence on higher education and its functions. The vital role of curriculum for higher education caused the formation of an important research in the field of MOOC's effects Curriculum in higher education systems in different countries. This issue looks very important; especially in Iran that strongly concerned about development and looked forward in the way of the mission of higher education and curricula which provided Twenty-Year perspective realization.

MOOC, not only could reach an important role in the world of teaching and learning as an online training tool, but also knew as an intellectual wave which attracted many authors and researcher attention. Although this issue is doubtful in Iran, because not only MOOC is not used as the most important tool, but also there is still no research about this issue.

\section{Theoretical Foundations and Background}

In the world of online education, Acronym "MOOC" is not a new principle, this term was proposed on online training course in Connectivism and Connective Knowledge Discussion from the University of Manitoba in 2008. Looking for nature of the pervasive training program, this university make MOOC courses available for audience (Students were free to join the training course) and unbelievably, more than 2000 students registered in these courses (Kathy, 2013).

MOOCs are an online learning environment in which you could have pleasant experiences by holding these courses (Salmon, 2013). Like to get the opportunity to participate in laboratories-discussions-meetings in low-cost or even free (Pappano, 2012). These courses are designed to Promoting and supporting a large number of students in informative and strategic way and it shows Significant results from the discussions and reports and calculations. In the past few years MOOC affected traditional teaching methods, online and widespread. Increasing the use of technology in order to apply teaching methods would challenge traditional notions of teacher and learner engagement. It seems that MOOC is another source for teaching and learning, while we could say that it has much more appeal than other techniques (Parry, 2013). As there is no complete way of training, MOOC has some Bugs and defects too, and it is suitable for those who are interested in learning a particular field and don't want to get certain Certificate. There is always a doubt for replacing Face to face training in universities and colleges with MOOCs (Kirschner, 2012). 
Most of MOOCs designed for short courses for adults, up to now. This makes courses available and Favorite for a wide range of learners (Koller, 2012). The initial wave providing courses on topics for staff and students, coordinated developments in online technology and with limited Support-Typically focused on computer science and other technology. However, details of courses are covering a wide range of topics and Coursera plans (Parry, 2013).

Parker also expects future versions of the MOOC platforms allowed to form small directed groups by Students properties (Parker, 2014). Changes and moves in MOOCs are toward self-manage in which students could say "I know how to do it". For example, "I can do your statistics or data analysis and my MOOC-based Certificate shows that". Students could improve their learning experience with engaging in certain types of MOOC-based courses and attend in some cases that lead to certification course (Kirschner, 2013).

Four competences could be effective in continuing to learn and participation in MOOC-based courses that contained Knowledge of English, Prior knowledge on the subject, Thought range, Self-regulation and self-efficacy, and communication skills (Abeer \& Barak, 2013). Student participation also could be effective by four features which obtained MOOC Value that contained Clear explanations, visualize abstract concepts, support and communications, and a variety of assignments for students who had Lack of information and basic qualifications for entry. Otherwise, there is possibility of leaving by students during courses even if organizers designed MOOC well. Similarly, highly qualified students, in learning in bad-designed MOOC, probably might fail at the end of terms (Abeer \& Barak, 2013).

The role of teachers in MOOC-based curricula contained answer the questions and discussions and public debates, solve the problems that students do not understand in video lectures, send a message to encourage students to participate in the forums, advise and encourage students to read literature and resources, send e-mail and announcements about the courses (Abeer \& Barak, 2014). Analysis show that the role of teacher in MOOC is different from his role in traditional courses, because these course need to deal with the massive number of students from different cultures.

MOOCs should move toward a developed model to encourage universities to create the course for free. Probably the thing that should happen is some charities pay the price of courses. In some cases, MOOCs should develop commonly with the country in which will be used. It should also be in indigenous language. It also should not be done only by the most famous faculty and it might be done by Faculty of Education or College of Teachers (Mintz, 2015). Ben Daniel represented "University education is reaching the point of decreasing returns, because staffing costs is growing unbearably and the necessary changes must be provided (Daniel, 2012). While it hardly seems that MOOCs presented a complete cure for "Cost disease" of higher education (Bowen, 2012), many identified ways in which MOOCs might affect the current economic model of higher education in United States and outside that. Kalbaugh in Wake Technical College believes that MOOCs could play the role of University of opportunities to learn about low-cost, low-risk and perpetuity more affordable and give this opportunity to adults who are not looking for certificate (Kalbaugh, 2014). Colleges and universities might continue to provide fundamental skills and knowledge, but the flexibility of online learning provide learning experiences "just in time" during a career for a person (Acemoglu, Laibson \& List, 2014). About the impact of MOOC on higher education, Billington and FranMueller (2013) presented the possible scenarios in terms of its effects on higher education:

$>$ MOOCs overcomes higher education in many universities.

$>$ MOOCs are Bubbles, Stopped and disappeared.

$>$ Universities could use MOOCs as Complement to their classes.

$>$ Universities will be Supervisors of MOOC tests.

> Universities Collaborate with MOOC.

$>$ MOOC give official permit of universities.

$>$ MOOCs are the key tool for competency-based training. 
Peercy, and Cramer noted that there is severe shortage of academic members for fulfilling rising demand for engineering documents in University of Wisconsin-Madison and he believes that MOOCs could play an important role in working on this concern (Peercy and Cramer, 2011). Similarly, Mintz from University of Texas, predicted the developed-MOOC could handle unmet needs for higher education in developing countries (Mintz, 2014).

There is valuable research about MOOC in higher education in developing countries. In a study on 83 learners who participated in MOOC courses that held by CCKO8 in title of "Connectionism and Linking knowledge", Finy (2009) Concluded learners though that certificate is not enough to motivate them for completing online course. In this regard, Mac Nice (2010) in his research studied on comments of 23 people in MOOC courses in title of "Connectivism and Connective Knowledge" which held by MIT University. The results of their study shows that learners had different percent of satisfaction because of options to choose training routines. Some of them like autonomy cause they knew that they could do anything they want, without criteria for evaluating and grading hindered their work. Some of them strongly needed guidance from teacher and some believed interaction between classmates were weak.

Koop (2011) in a research on 55 learners that participated in MOOC courses which was held by PLENK in title of "Critical writing courses and personalized learning, networks and knowledge contexts", found out 77 percent of students believed that provided sources like daily emails which related to courses were enough for understanding the course even before the start. Participants were satisfied with gathering and sharing sources together or be familiar with them, but some of them launched to create digital media like blogs and the majority was over 49 years. In another study, Breslow (2013) in a research on 7161 learners that attended in MOOC courses was held by (edX) in title of "electronic circuits" and participated in survey of research, represented that the goal of leaners company was the acquisition of knowledge and a higher chance of proposed work, or a personal challenge to evaluate their ability with MIT courses. $90 \%$ activities belonged to learners who had active participation and observed the posts. There is no relationship between age, gender or individual's motivation or success. However, learners who had offline work with someone, had higher scores than who act alone.

Goh, Kaur \& Chion (2014) in study in title of "Perceptions of MOOC based on activity theory" in 160 Malaysian students found out that most learners insisted on usefulness of MOOC courses in their learning and they claimed that their learning is enhanced. They were satisfied with MOOC courses and advised them to their Counterparts. Overall, students believed that MOOC courses could be an effective process to complete traditional teaching methods. Ulrich and Nedelcu (2015) in a study entitled "MOOC in our university: Hopes and fears", perused the effects of MOOC courses on students participating in the courses of the University of Bucharest. The main question posed in this study is that how we could transform a new trend of training like MOOC to realistic and inspiring process? The method of this research was a case study in comprehensive university in Hungary in which they data collected through content analysis, interviews and online questionnaires. Research community included 67 undergraduate students of Faculty of Psychology and 33 members of the faculty. The findings indicate that MOOC's expectations are unrealistic and they are not aware of their duty in their field. The feel of disability must be eliminated, too. It will be shared as syndrome among the academic communities.

Mercado and et al (2017) in study in title of "Connectivity of Learning in MOOCs: Facilitators' Experiences in Team Teaching" in 135 facilitators in hybrid courses (cMOOC + $x M O O C$ ) found out that the collaborative construction of knowledge is the most widely used strategy to promote learning connections in MOOCs and that its design is the biggest challenge that facilitators faced while carrying out their activities. In another study, in title of " Community trscking in a CMOOC and nomadic learner behavior identification on a connectivist rhizomaticle arning network" Bozkurt and et al (2016) studied the literature on connectivism, connectivist MOOCs (cMOOCs) and rhizomatic learning by 
examining participant interactions, community formation and nomadic learner behavior in a particular CMOOC, \#rhizo15, facilitated for 6 weeks by Dave Cormier. As an explanatory mixed research design, Social Network Analysis and content analysis were employed for the purposes of the research. SNA was used at the macro, meso and micro levels, and content analysis of one week of the MOOC was conducted using the Community of Inquiry framework. The macro level analysis demonstrates that communities in a rhizomatic connectivist networks have chaotic relationships with other communities in different dimensions (clarified by use of hashtags of concurrent, past and future events). A key finding at the meso level was that as \#rhizo15 progressed and number of active participants decreased, interaction increased in overall network. The micro level analysis further reveals that, though completely online, the nature of open online ecosystems are very convenient to facilitate the formation of community. The content analysis of week 3 tweets demonstrated that cognitive presence was the most frequently observed, while teaching presence (teaching behaviors of both facilitator and participants) was the lowest.

Overall we can say Studies about MOOC, although it is a new phenomenon, each year followed an ascending line and the number of research that has been assigned to it, was impressive. the goal of this research is validation of MOOC-based curriculum model in higher education in Iran. Answering the following questions were in the agenda:

1) What is the proper model of MOOC-based curriculum model in higher education in Iran, from the perspective of educational technology experts and technology higher education?

2) Which Credit did designed model of MOOC-based curricula in higher education have?

\section{METHODOLOGY}

The present study is a qualitative and grounded theory research. The research method is mixed and of exploratory type, which consists of two major qualitative and quantitative stages. In the qualitative stage, a semi-structured interview tool was used and in the quantitative stage a researcher made questionnaire was used (Creswell, 2004). In an exploratory plan, a measurement tool will be developed usually through a qualitative research. In the present study, quantitative measurement tools have been developed through qualitative study. The researcher first collects the required information through the qualitative research method. The collection of qualitative information leads to the description of many aspects of the phenomenon. This basic knowledge allows for the formulation of a hypothesis about the occurrence of the phenomenon under study. After this stage of research, in order to test the developed hypotheses, the researcher can validate the data from the qualitative stage using quantitative methods and then modify, replace or eliminate the factors that were less effective (Bazargan, 2010).

The grounded theory also provides an abstract analytical framework of a process. Participants in this study must all have experienced the intended process and the elaboration of this theory can be useful in explaining their mode of action, or provide a framework for future research (Creswell $I, 2014$ ). Accordingly, the grounded theory is a qualitative research project in which the researcher provides a general explanation (or a theory) of a process, action, or interaction which is the result of many participants ' views (Strauss \& Corbin, 2015). The grounded theory is performed during the open, axial, and selective coding process and in the form of a paradigm consisting of underlying conditions, causative conditions, mediator conditions, Central phenomenon, outcomes and strategies. In the present study, due to the subject of research and due to its novelty in Iran, the grounded theory has been used in order to design a model for a MOOC-based curriculum in higher education in Iran, as well as identifying and understanding the factors involved in this process, and also providing an abstract analysis of each of the components and the relationships between them. 
Participants and Sampling

The statistical population of the study consists of two groups. The first group is composed of experts in the field of educational technology and higher education technologies throughout the country. These people have executive records and are experienced in attending these courses. The second group consisted of faculty members and Ph.D. students in educational sciences sub-disciplines in the academic year of 2016-2017, in public universities of Tehran where offered a PhD degree in educational sciences. This group consists of 476 people, including 90 faculty members and 386 PhD students.

In the first group, selected samples were individuals who had the experience of participation in or implementation of these courses, who can introduce the components of the MOOCs, based on which, they will provide accurate comments on the quality of the educational services in these courses. Therefore, in the present study, the criterion of membership in the sample group for the first group of people is to have such an experience at the time of the research. In this research, the sample size has been determined in a qualitative way through targeted sampling. Sampling continued until data saturation, and by interviewing the 14th person, theoretical saturation in the categories was achieved. As a result, 14 people were identified as the sample size in the qualitative section. These people also have significant publications and research activities in the field of educational technology, as well as management and executive background in the field of educational technology in higher education.

The second research community composed of the faculty members and Ph.D. students of the educational sciences (all sub-disciplines) of the public universities of the Ministry of Science where offered a PhD degree in educational sciences in Tehran. This group comprised of 476 people (386 students and 90 faculty members). From this population, 214 people were identified as sufficient sample size using Morgan Table. Subsequently, using proportional stratified sampling, questionnaires were distributed among the statistical sample group. In proportional stratified sampling, the sample size is proportionate to the population size (Gull et al., translator Nasr et al., 2004). In this research, a number of members of each university were selected for the sample population according to the size of the population of each university in the statistical population, and research questionnaires were distributed among them.

\section{Tools/ Instruments}

In the present study, semi-structured interview was used in the qualitative section which included a total of 34 questions about: strengths, weaknesses, opportunities and threats of MOOC for higher education curriculum; facilitating factors in resolving weaknesses and threats; fortifying factors in realization of strengths and opportunities; Reasons and philosophy of designing MOOC -based curriculum in higher education; Objectives, content, teaching- learning method and evaluation of MOOC-based curriculum in higher education; Mock-based curriculum strategies in higher education; as well as the consequences of a MOOC-based curriculum. The validity of qualitative data was reviewed by five non- attendant specialists. And its reliability was calculated 0.81 through intercoder reliability (two coders) (Miles \& Huberman, 1994).

\section{Data Analysis Method}

In the qualitative section of the present study, open, axial and selective coding method for analyzing the interviews has been used. In this regard, first, in open coding, verbal evidence, basic concepts, and categories were extracted, which were considered as the basic findings in the subsequent coding stages of the research. After the open coding and extracting the categories and also after the axial coding and finding the relationship between the categories in the form of paradigm components (underlying conditions, causative conditions, mediator conditions, Central phenomenon, outcomes and strategies), the next step was selective coding and interpretation of relationships between categories. In the quantitative part, using raw scores from a questionnaire in a range of 1 to 5 for each response, a conceptual model for measuring the interaction between structures was obtained from the correlation calculation method (SPSS), path 
analysis, and structural equation modeling (AMOS software). So, firstly, the relationship between variables is calculated by Pearson correlation method and then, by path analysis and structural equation modeling, the conceptual model of the research is more precisely calculated. By modifying the model and calculating the final fitted model, the proposed model for the study of the process dimension of the MOOC-based curriculum was presented. In this stage, descriptive statistics were also used. To determine the fitting of models in AMOS software, the fitting indices recommended in Yalaha were used (2000: 452).

\section{RESULTS}

\section{Question 1}

What is the proper model of MOOC-based curriculum model in higher education in Iran, from the perspective of educational technology experts and technology higher education?

\section{Open coding phase: 930 Proposition initial concept}

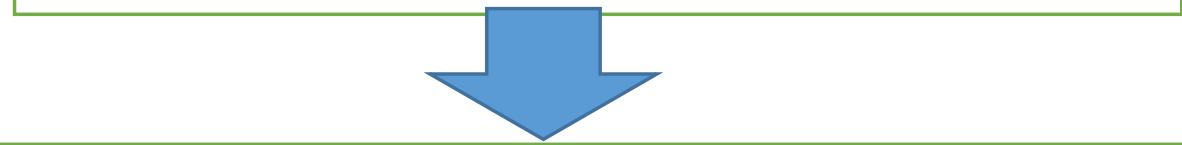

Axial coding phase: 28 general categories

A) Causes terms: 1- Emphasis on active learning; 2- Attention to individual differences and intrinsic interest of learners; 3- Expand interaction between teachers and learners; 4- Achievable goals; 5Emphasis on the exchange of information and increase the range of people's information; 6- Using various and multiple resources; 7- The feedback of the curriculum

B) Central phenomenon: MOOC-based curricula in higher education: 1goal; 2- Content; 3- Procedures; 4- assessment

C) Underlying conditions: 1- Strengths; 2- weaknesses; 3Opportunities; 4- threats

D) Confounding terms: 1-combination of MOOC-based curricula and traditional training; 2- Professional ethics and Professional principles are utilizing MOOC; 3- Creating a culture of learning MOOC and the necessity of using them in the curriculum; 4- increased support from universities for MOOC; 5- Active participation of experts in the field of education and e-learning in designing MOOC

E) Strategies: 1- effective curriculum for MOOC; 2- Increase international relationships in higher education; 3- Social and cultural infrastructure for MOOC utilization; 4-have Strategic planning for MOOC in the field of higher education; 5- Apply good mechanisms of management for MOOC utilization

F) Implications: 1- Dynamic curriculum of higher education; 2- the possibility of reloading efficient education structure and university curriculum; 3- the possibility of Expansion of international relations in the curriculum of higher education

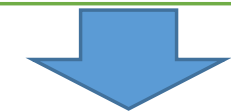

Selective coding phase: 6 dimension of paradigmatic model

Causes (7 Categories); Central phenomenon: MOOC-based curricula (4

Categories); Underlying conditions (4 Categories); Confounding terms (5 Cateqories); Strateqies ( 5 Cateqories); results ( 3 Cateqories)

Figure 1. The process of coding and data reduction 


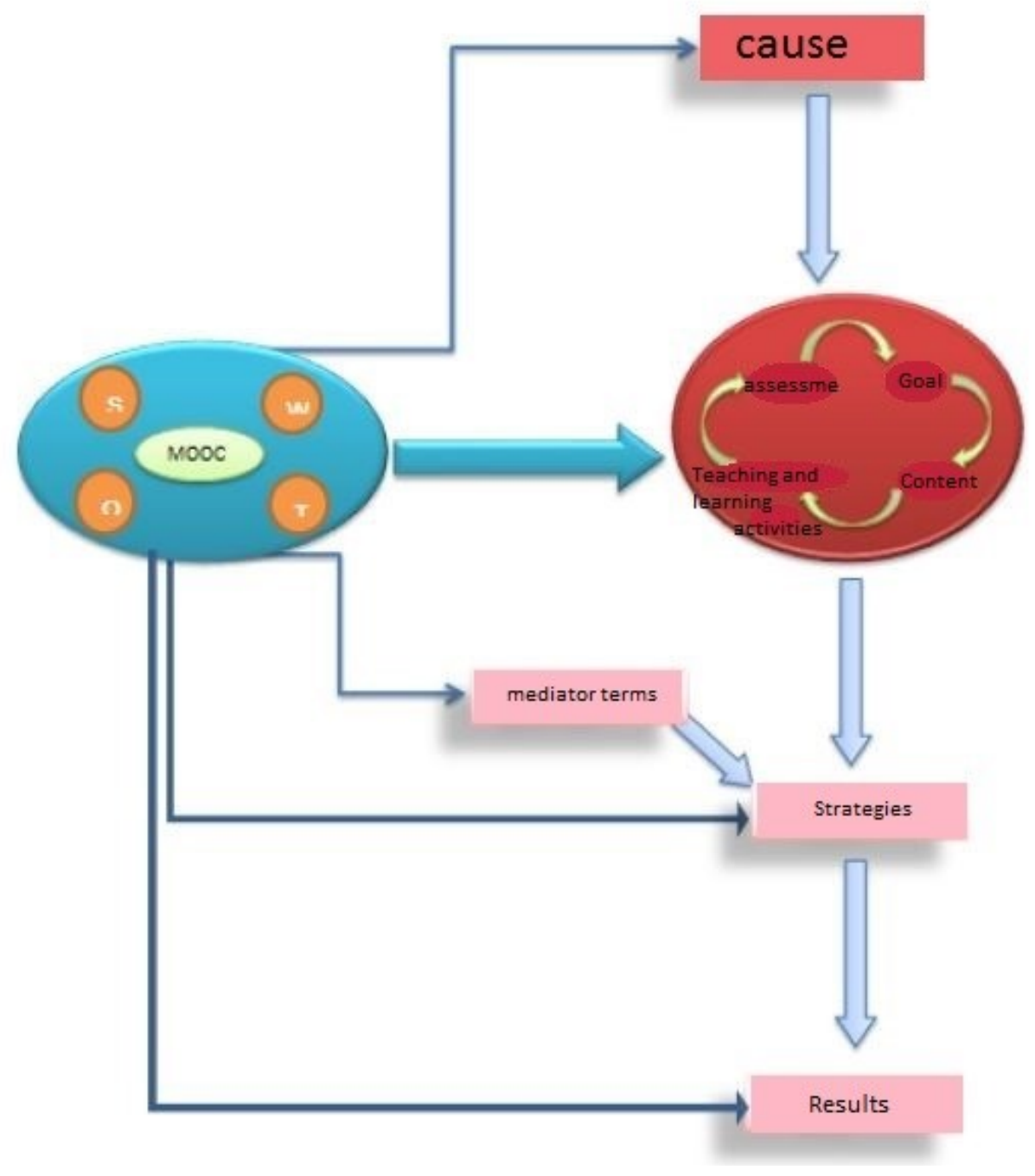

Figure 2: Strauss and Corbin paradigmatic model in study

In order to answer this question, through interviews with experts, major and minor categories about MOOC-based curricula in higher education is identified and the final conceptual model codified. The following chart shows trends in data reduction and the number of open code, axial and selective categories, it represented The paradigmatic model. In fact, the grounded theory approach, Sub-categories and features of each one related to the central issue which included causes, central phenomenon, Underlying conditions (environmental), confounding terms (mediator), Strategies and Implications.

\section{Question 2}

Which Credit did designed model of MOOC-based curricula in higher education have?

According to findings of the qualitative research obtained from paradigm pattern, hypothetical model is considered. Then, after removing non-meaningful routes, due to its compatibility with the findings of the qualitative analysis, satisfying model estimation indices and being meaningful statistical estimates are considered for provided data. 
Table 1. Descriptive statistics and Pearson correlation coefficient based on the dimensions of the phenomenon, strategies, and outcomes

\begin{tabular}{|c|c|c|c|c|c|c|c|c|c|c|c|}
\hline & 1 & 2 & 3 & 4 & 5 & 6 & 7 & 8 & 9 & 10 & 11 \\
\hline 1.Strength & - & & & & & & & & & & \\
\hline 2. Weakness & $0 / 168$ & & & & & & & & & & \\
\hline 3.Opportunity & $0 / 584$ & $0 / 649$ & & & & & & & & & \\
\hline 4. Threats & $0 / 525$ & $0 / 637$ & $0 / 785$ & & & & & & & & \\
\hline $\begin{array}{l}\text { 5.Causes } \\
\text { Terms }\end{array}$ & $0 / 399$ & $0 / 500$ & $0 / 510$ & $0 / 609$ & & & & & & & \\
\hline $\begin{array}{l}\text { 6. Mediated } \\
\text { Terms }\end{array}$ & $0 / 401$ & $0 / 453$ & $0 / 519$ & $0 / 626$ & $0 / 879$ & & & & & & \\
\hline 7.Goals & $0 / 483$ & $0 / 526$ & $0 / 609$ & $0 / 635$ & $0 / 729$ & $0 / 656$ & & & & & \\
\hline 8. Content & $0 / 393$ & $0 / 424$ & $0 / 519$ & $0 / 572$ & $0 / 624$ & $0 / 643$ & $0 / 596$ & & & & \\
\hline 9.Teaching & $0 / 376$ & $0 / 453$ & $0 / 466$ & $0 / 524$ & $0 / 511$ & $0 / 494$ & $0 / 512$ & $0 / 671$ & & & \\
\hline $\begin{array}{l}10 . \\
\text { Assessment }\end{array}$ & $0 / 275$ & $0 / 283$ & $0 / 406$ & $0 / 476$ & $0 / 466$ & $0 / 533$ & $0 / 437$ & $0 / 602$ & $0 / 513$ & & \\
\hline 11. Strategies & $0 / 520$ & $0 / 498$ & $0 / 646$ & $0 / 774$ & $0 / 567$ & $0 / 657$ & $0 / 601$ & $0 / 545$ & $0 / 437$ & $0 / 551$ & \\
\hline $\begin{array}{l}12 . \\
\text { Consequences }\end{array}$ & $0 / 397$ & $0 / 458$ & $0 / 476$ & $0 / 522$ & $0 / 790$ & $0 / 694$ & $0 / 550$ & $0 / 534$ & $0 / 442$ & $0 / 446$ & $0 / 567$ \\
\hline
\end{tabular}

As Table 1 shows, all relationships between variables in conceptual model were positive and meaningful; $\min =0 / 275, \max =0 / 879, M=0 / 540, S D=0 / 115$. For a more accurate calculation model, estimation calculated with Structural equation modeling in the study sample data. According to proposed corrective measures in AMOS software, Conceptual Model corrected for achieving higher estimation levels.

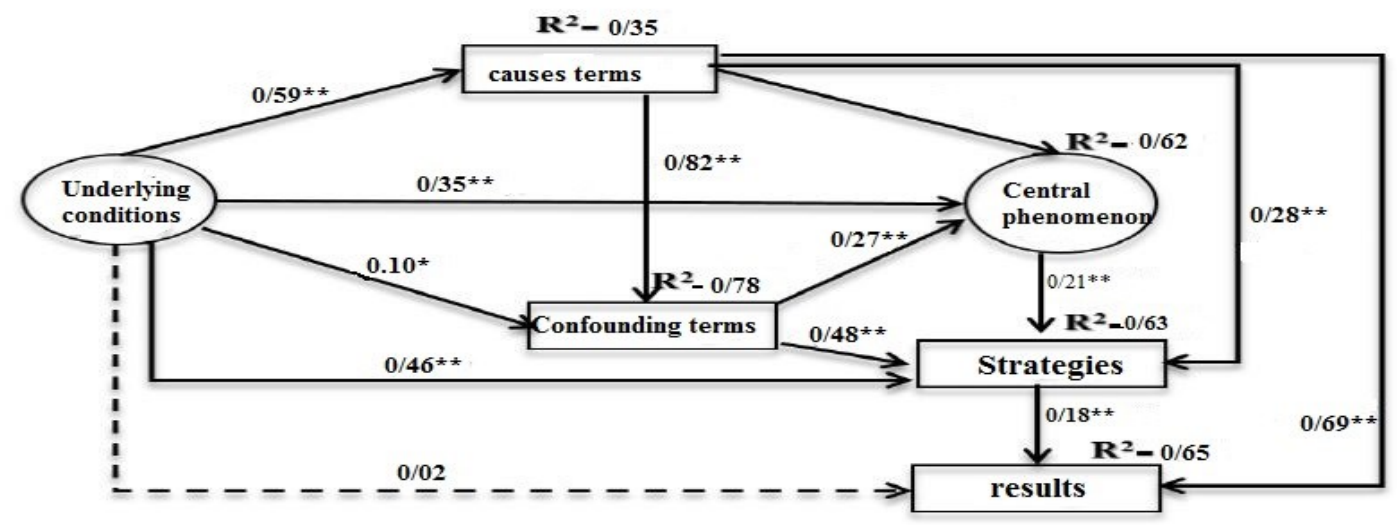

Figure 3. Estimated conceptual model

Note: *: Coefficients were significant at levels of alpha $0 / 01$ to $0 / 05 ; * *$ : Coefficients were significant at smaller levels of alpha 0/01; Solid lines represent meaningful routes and Dots represents routes in the conceptual model; Errors and dimensions of latent variables didn't draw to avoid the complications. 
As specified in Figure 3, straight path of Underlying conditions has not shown statistically significant results. On the other hand, causes terms mediated relationship of central phenomenon with underlying conditions, Confounding terms, Strategies and results. Central phenomenon in spite of impress from underlying conditions, causes terms and Confounding terms, in mediation impact of these variables on strategies had significant role. As you can see results were mostly impressed directly by causes terms. Causes terms also related indirectly to underlying conditions and results $(b=0 / 471, p<0 / 05)$. Finally, Strategies just had $0 / 18$ relation with results.

Table 2 Shows The results of the Estimating of Figure 2.

\begin{tabular}{|c|c|c|c|c|c|c|c|c|c|c|c|c|}
\hline & CMIN/DF & $\mathbf{P}$ & SRMR & GFI & AGFI & NFI & RFI & IFI & TLI & CFI & RMSEA & PCLOSE \\
\hline $\begin{array}{l}\text { Allowable } \\
\text { Amount }\end{array}$ & $5>$ & $>0 / 05$ & $0 / 08>$ & $0 / 90 \leq$ & $\leq 0.90$ & $\leq 0.90$ & $\leq 0.90$ & $\leq 0.90$ & $\leq 0.90$ & $\leq 0.90$ & $0 / 08>$ & $>0 / 05$ \\
\hline Observed & $0 / 936$ & $0 / 422$ & $0 / 007$ & 0/996 & $0 / 970$ & 0/997 & $0 / 987$ & $1 / 000$ & $1 / 000$ & $1 / 000$ & $0 / 001>$ & $0 / 628$ \\
\hline
\end{tabular}

As Table 2 shows, all estimating indicators were within the allowable range. Thus conceptual model after correction showed good estimation with data. The corrections included removing direct route to the consequences of the underlying condition; added direct route from causes terms to confounding terms, Strategies and results; and adding direct route of confounding terms to Central phenomenon.

\section{DISCUSSION AND CONCLUSION}

Nowadays, increasing the volume of knowledge and information, rapid Obsolesce of lessons, rapid changes in societies and unpredictability of the future, needs for education and continuous learning instead of Sectional training. In this regard, efficient and effective use of educational technologies contributed in lifelong learning and facilitate its realization. Therefore, the importance and necessity of putting together comments of experts are a way for using new, critical and important technologies so that we can achieve the maximum benefit from new educational technologies like MOOC.

As revealed in this study, an important strategy in MOOCs is developing efficient curricula. While technical and scientific infrastructures are necessary, learning and teaching are not possible without efficient performance of the curricula. This means that online curricula in general and MOOCs in particular need constant revision in terms of plans and ceaseless accumulation of knowledge by, not only the professors but also, the planners. It is easy to understand from the results that any useful performance requires scientific, technical provisions and facilities and teachers who promote their knowledge along with these novel technologies to ensure high quality teaching. All these requirements are possible with a proper planning and an efficient organization of the curricular elements.

Another major strategy in applying MOOCs in curricula has to do international relations in higher education. This is particularly significant considering the need to keep up with present level of knowledge by developing countries like Iran. In fact, higher education plays a vital role in national development since it is responsible for creating innovations and training committed educated human power for various sections. This development is realized only if higher education undergo radical reforms and its activities attain international standards. Professors and curricular planners must base their efforts on this and lead students toward the goals, a success achieved by excellent supervision and management of higher education and planning curricula according to international criteria and effecting proper changes in knowledge, skills, and attitudes of learners. International criteria such as increased interactions between professors and academia worldwide, following pioneer excellent campuses, emphasizing international educations, etc., rely on strengths and performance of professors and officials. 
Socio-cultural preparation for using MOOCs is another strategy in applying MOOCs in curricula. Socio-cultural contexts in higher education must be shaped in order to be able to accommodate novel attitudes. Despite being free of charge, MOOCs do not consider local culture and milieu and this makes them difficult to understand for oversea learners (Obeer and Barak, 2014; Chafkin, 2013).

Through MOOC-based curricula, planners, designers and managers have access to communication technology, new tools and methods. Thus, conventional teaching methods change and users encounter vast spectrum of info and knowledge. Wrong perceptions and misunderstandings among professors affect the application of MOOCs in curricula. No educations tale place in void. The education including MOOCs takes place in the social context of the life of students and professors, both influencing the environment and influenced by it. This reciprocity has more to do with culture. Culture does shape the beliefs and attitudes of professors and students. This means that an open flexible culture encourages professors and students to cultivate positive views toward new technologies.

Strategic plans in application of MOOCs in higher education is another major strategy. Higher education in Iran has considerable growth recently and has brought about radical transformations in a number of scientific fields. Higher education in Iran is about to enter to a new era thanks to new technologies like MOOCs. One major challenge for this higher education is to sustain self-sufficiency and financial reward while offering high quality education in form of MOOC-based curricula. Thus, it is necessary to develop vivid strategies to attain this. Offering MOOCs makes higher education in Iran define and justify its audience, value, and e-commerce. In other words, strategic planning is a way to effect the roles and actualize the goals of higher education. Strategic planning is not limited to designing MOOCs and applying them in higher education but is a tool to determine the multiple grand policies in higher education and to reform and strengthen them. This has to do with harmonizing various activities of various sections in higher education and developing capacities and opportunities based on institutionalizing MOOCs in higher education.

One of the most basic strategies that determine efficient application of MOOCs in higher education is proper management of optimal use of MOOCs. In order for higher education to develop by exploiting new technologies like MOOCs and in the shortest time possible with the least expenditures, all national and local resources and facilities must be arranged accordingly. Here, a comprehensive considerate perspective toward human/non-human aspects can be useful. Therefore, identifying and employing all factors, potentials, capacities and abilities within higher education stands as the primary effort in efficient application of MOOCs. Human and societal elements, on one hand, and exploiting all local, social, and cultural capacities and infrastructures and supports, on the other hand, are important. Identification of these capacities both helps planners and developers and helps specify the proper kinds of businesses in higher education as a primary step.

All in all, it is fair to say that these strategies are vital to an effective application of MOOC-based curricula in higher education and to get positive results. In what follows, three chief consequences of MOOC-based curricula in Iran are explained: One consequence that research subjects noted is the increased dynamism in collegiate curricula. Downs (2008) stresses flexible educational experience for learners. Flexibility created by MOOCs allows students to use them wherever accessible (Levi, 2011). This initiates a Feedback cycle. Another consequence that present paper anticipates for MOOCbased curricula has to do with increased international ties in higher education. As Potter (2013) understands it, MOOCs plays a significant role in making education international and developing oversea educational projects.

An effective revision of education system and collegiate curricula is another consequence of higher education curricula examined in this study. With emergence of MOOCs and the shift from qualification by degree to qualification by institutions producing MOOCs, a 
reconsideration of education system and educational quality is inevitable. This hopefully means more efficient education.

This research led to design the model of MOOC-based curricula in higher education according to grounded theory. MOOC-based curricula as a central issue that other Components (Causes terms, Central phenomenon, Confounding terms, Strategies and Implications) Organized with its centrality. The mission of these components is help for realization elements of curriculum. According to conceptual research model, elements of MOOC-based curricula in higher education included purpose, content, teaching-learning methods and assessment which are in a cyclical relationship with each other. The elements of curriculum are based on causes terms and has some results. In fact, effective realization of elements and their relationship with each other is possible through results; Terms of intervention affected this process. These terms and procedures had results in higher education. All these interactions happened in situation in which strengths, weaknesses, opportunities and threats prepared necessary condition for it.

The model obtained in this research is a feasibility study on implementation of MOOC in Iran's higher education system. Aspects and the possibility of realization and implementation of it have been examined from the perspective of experts. The point to be addressed is that in addition to the designers view, the users' views are very essential. Students' views as key users of MooCs play an important role in the successful implementation of MOOC and models of this kind. In this regard, we can refer to a model in the field of MooCs designed by Xiao and Pardamean (2016) in Indonesia, which is consistent with the needs and demands of both developers and users.

On validating model of research in order to determine the amount and nature of that relationship, it is shown that central phenomenon affected by causes terms, confounding terms and has medium role on the impact of these variables on strategies. This indicates that Central phenomenon which is formed by elements of MOOC-based curricula, has a pivotal role in the model that matched with its mission in Strauss and Corbin paradigm pattern (2016). In this pattern, Central phenomenon is an axis that other elements and their relation organized by it and around it. Furthermore, it was observed that results mostly affected from causes terms. Probably it is because determine the logic of curriculum at the time of its design, is very important and it could obtain curriculum's path and its results. This importance become clear when we see in estimated model, causes terms are mediator in connection between Underlying conditions and Central phenomenon, mediated conditions, strategies and results. The validity of the findings, relations between components shows that MOOC-based curriculum take place during the process of action / interaction among components. Actions and relationships between model components, represents a systematic plan that should be considered in any type of MOOC-based curriculum. Determine the severity of the impact and effectiveness of each component of the model could be helpful in decision-making and process design in the curriculum of higher education administrators and planner's curriculum.

We should consider some important points in using MOOCs; that there is not The growth and assimilation in developing countries in comparison with developed countries in MOOCs and these countries in the near future according to the spread of MOOC in education, would suffered from challenges posed by the import of it. This issue for all developing countries is highly likely and inevitable and Iran also is not an exception. The continuation of this problem will form a new colonialism in the world which in this time, shown in the form of training and education of the younger generation that is far more dangerous than the old colonial. Therefore, it is necessary to perform Indigenous production and sharing global production and stay ahead in this area, along with utilizing MOOCs in higher education and Took advantage of its benefits. 
At the end, we mentioned Operational research proposals which include:

$>$ The proposed conceptual model in research could be paced as a basis for the development of MOOC-based curriculum in University of Tehran and other centers of higher education.

$>$ Curriculum inferred from this study performed in one of the Public Universities of Tehran, for trial.

$>$ We recommended to directors and administrators of universities and centers of higher education to launch necessary support as mediator terms which obtained in this research.

$>$ Based on the results of this study, it is very important to consider underlying conditions and to plane for strengthen and optimize its procurement, as Conditions affecting all components and factors involved in the MOOC-based curriculum design.

$>$ According to the crucial role of causes terms in the MOOC-based curriculum in this study, legitimizing and Justifying the beneficiaries of higher education in the design and implementation of such program, would help Enhance functionality and effectiveness.

$>$ According to the regulation authority in compiling and reviewing the curricula of universities having an audit board, we suggested that MOOC Designed Curriculum Within each university perform with the participation of educational technologists, faculty, planners and administrators in it. These partnerships inside the university in Codifying MOOC-based curriculum, reduce possible resistance that could happen by some people within the university and facilitate MOOC curriculum in university.

\section{BIODATA and CONTACT ADDRESSES of AUTHORS}

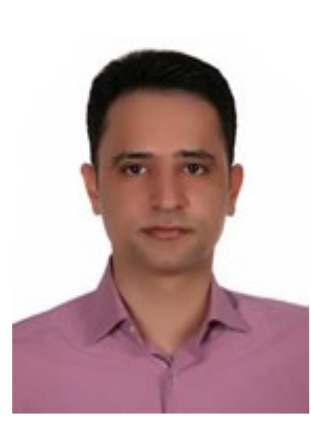

Esmaeil JAFARI is an Assistant Professor of Education, Department of Technological Learning, Shahid Beheshti University, Tehran, Iran. Dr. Jafari gained his Ph.D. in Higher Education Curriculum in February 2017. His academic interest areas are MOOCs, Knowledge Management, Virtual Curriculum, Virtual Reality, Educational Entrepreneurship, E-learning and comprehensive research on virtual education. He currently serves as an organizer and member of the Executive Committee, Iran's National Elites Foundation. He has over 10 journal articles published in national and international indexes, 1 international book chapter, 6 technical books and other national and international articles and papers submitted to national and international conferences.

Dr. Esmaeil JAFARI

Department of Technological Learning,

Shahid Beheshti University,

1983969411, Tehran, Iran.

E-mail: phd_136287@yahoo.com

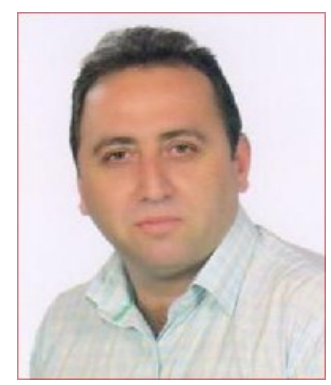

Kourosh Fathi VAJARGAH is a Professor of Curriculum Planning and Educational Managment at Faculty of Education and Psychology, SBU, Tehran, Iran. Dr. Fathi gained his Ph.D. from Tarbiat Modarres University and then completed specialized and technical courses on Educational Management and higher education planning at Universities such as Cassel University, Germany, Southern Crescent University and Griffith University, Queensland, Australia. His academic interest areas area educational needs assessment, human resources education and training, E-learning, higher education curricula and distance learning. He has over 100 journal articles published in national and international indexes, 30 national international book chapters and other national and international articles, papers submitted to international conferences in the area of E-Learning and higher education. 
Dr. Kourosh Fathi VAJARGAH

Faculty of Education and Psychology

Shahid Beheshti University, 1983969411, Tehran, Iran.

E-mail: k-fathi@cc.sbu.ac.ir

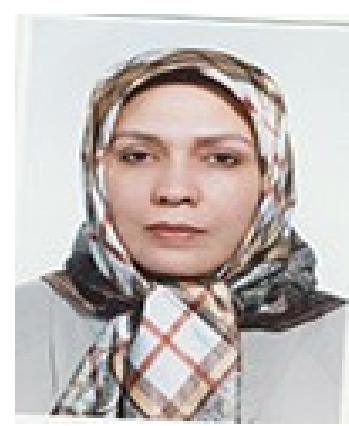

Mahboobeh AREFI is an Associate professor of Curriculum and Higher Education at Faculty of education and psychology, SBU. Dr. Arefi gained his Ph.D. in Curriculum Planning from Tarbiat Modarres University, Tehran, Iran. Her academic interest areas are learning and teaching process, curriculum planning, higher education, educational evaluation, education futures, e-learning and use of internet in education. She has over $\mathbf{2 5}$ journal articles published in national and international indexes, 4 national books and chapters of 2 books and other national and international articles, papers submitted to international conferences.

Dr. Mahboobeh AREFI

Faculty of Education and Psychology;

Shahid Beheshti University, 1983969411, Tehran, Iran.

E-mail: M-Arefi@sbu.ac.ir

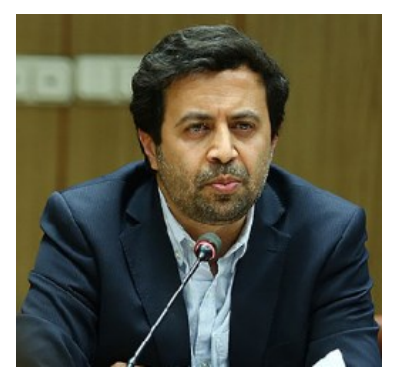

Morteza REZAEIZADEH got his PhD in e-learning and entrepreneurship from University of Limerick in January 2015. His PhD thesis was on using a collective intelligence methodology to design an e-learning platform for cultivating students' core entrepreneurial competencies. He is a member of International ELearning Association (IELA) located in New York, USA. Morteza has attended many international conferences in the area of e-learning and has been awarded the Best Paper award in "2013 International Conference on Information and Education Technology" (ICIET 2013) held in Brussels on 12-13 January 2013. He is now an adjunct lecturer in the school of education, UL. His main research and teaching interest areas are the cutting-edge technologies which could be implemented in learning environments. He has already been involved in a European research project and is now working on a research project for designing an Augmented Reality application which could enhance teaching-learning experiences in primary schools' science books.

Dr. Morteza REZAEIZADEH

Department of Higher Education,

University of Shahid Beheshti,

School of Education, University of Limerick, Ireland

E-mail: Morteza.Rezaeizadeh@ul.ie

\section{REFERENCES}

Alonso mercado-vweela, M., Beltran, J., Villegas perez , M., , Rivera vazquez, N. \& Ramirrz-montoya, M. ( 2018). Connectivity of Learning in MOOCs: Facilitators' Experiences in Team Teaching, The Turkish Online Journal of Distance Education, 18(1): 143-156.

Abeer, W., \& Barak, M. (2014). "Students' preferences and views about learning in a MOOC." Procedia-Social and Behavioral Sciences, 152: 318-323.

Acemoglu, D., Laibson, D., \& List, J. A. (2014). Equalizing superstars: The Internet and the democratization of education. NBER Working Paper Series (19851). Retrieved from: http://economics.mit.edu/files/9452 
Bazargan, A. (2010). In troduction to qualitative and mixed research methods; Common approaches in behavioral sciences, Tehran: Nashr Didar(in Persian).

Beranek, L., \& Remes, R. (2012)."The course of e-commerce based on active learning." Proceedings of the 7th International Conference on Efficiency and Responsibility in Education (ERIE 2012).

Billington, P. J., \& Fronmueller, M. P. (2013). MOOCs and the future of higher education. Journal of Higher Education Theory and Practice, 13(3), 36-42.

Bozkurt, A., Homeychurch, S., Caines, A., Bali, M., Koutropoulos, A. \& Cormier, D. (2016). Community trscking in $A$ CMOOC and nomadic learner behavior identification on a connectivist rhizomaticle arning network, The Turkish Online Journal of Distance Education, 17(4): 4-30.

Bowen, W. G. (2012). The 'Cost Disease' in higher education: Is technology the answer? The Tanner Lectures at Stanford University. Retrieved from http://www.ithaka.org/sites/default/files/files/ITHAKATheCostDiseaseinHigher Education.pdf

Breslow, L. (2013)."Studying learning in the worldwide classroom: Research into edX's first MOOC." Research \& Practice in Assessment .8

Chafkin, M. (2013). "Udacity's Sebastian Thrun, godfather of free online education, changes course." Fast Company, 142013.

Creswell, J. W. (2004). Educational Research: Planning, Conducting, and Evaluating Quantitative and Qualitative Research. Pearson.

Creswell, J. W. (2014). Qualitative inquiry and research design: choosing among five approachs, $2^{\text {nd }}$ ed, translated by Hossein Danaiefard and Hossein Kazemi, Tehran: Entesharat Saffar (in Persian).

Daniel, J. (2012)."Making sense of MOOCs: Musings in a maze of myth, paradox and possibility." Journal of interactive Media in education 2012. 3

Downes, S. (2008). "Places to go: Connectivism \& connective knowledge." Innovate: Journal of Online Education 5.1

Fini, A. (2009). "The technological dimension of a massive open online course: The case of the CCKO8 course tools." The International Review of Research in Open and Distributed Learning, 10(5): 11-23.

Gall, M., Borg, W., and Gall, J. (2004). Quantitative and qualitative research methods in education and psychology. Translation by Ahmad Reza Nasr and others, Tehran: Samt(in Persian).

Goh, W. W., Kaur, S. , and Chion, Z. H. A. ( 2014). The Perceptions of MOOC among Learners Based on Activity Theory, presented in: Taylor's 7th Teaching and Learning Conference, DOI 10.1007/978-981-287-399-6_30

Jerry, B. (2000). The e-/earning potential. Retrieved from http://www.kdgonline.com

Khan, B. H. (2004). "The people-process-product continuum in e-learning: The e-learning P3 model." EDUCATIONAL TECHNOLOGY-SADDLE BROOK THEN ENGLEWOOD CLIFFS NJ- , 44(5): 33-40.

Kirschner, A. (2012). "A Pioneer in Online Education Tries a MOOC." Chronicle of Higher Education.

Koller, D.2012. "What We're Learning from Online Education." TED. Jun. 2012. Web.

Kop, R. (2011). "The challenges to connectivist learning on open online networks: Learning experiences during a massive open online course." The International Review Of Research In Open And Distributed Learning , 12(3): 19-38. 
Levy, D. (2011). "Lessons learned from participating in a connectivist massive online open course (MOOC)." Proceedings of the Chais conference on instructional technologies research 2011: Learning in the technological era.

Mackness, J., Sui M., and Roy W. (2010). "The ideals and reality of participating in a MOOC." 266-275.

McAuley, A., Stewart, B., Siemens, G., \& Cormier, D. (2010). The MooC model for digital practice.

Miles, M. B., \& Huberman, A. M. (1994). Qualitative data analysis; An expanded sourcebook ( $2^{\text {nd }}$ Ed. $)$. California: Sage publications.

Mintz, S. (2015). MOOC providers. Inside Higher Education, Higher Ed Beta 16 August 2015, insidehighered. Com/blogs/higher-ed-beta/mooc-providers, Accessed: 27 December 2015.

Ong, C. S., Lai, J. Y., \& Wang, Y. S. (2004). Factors affecting engineers' acceptance of asynchronous e-learning systems in high-tech companies. Information and Management, 41(6), 795-804.

Pappano, L. (2012). The Year of the MOOC. The New York Times. Retrieved from http:// www.nytimes.com/2012/11/04/education/edlife/massive-open-onlinecourses-are-multiplying-at-a-rapid-pace.html

Parker, C. D. (2014). "A descriptive study to identify deterrents to participation in employer-provided e-learning." 1-131.

Parry, M. (2013)."Competency-based education advances with US approval of program." The Chronicle of Higher Education

Peercy, P.S. and Cramer, S.M. (2011). "Redefining quality in engineering education through hybrid instruction", Journal of Engineering Education, Vol. 100, No. 4, pp. 625-629.

Potter, C. (2013). F is for failure; or, don't invest your Pension in MOOCs yet. The Chroni cle of Higher Education (July 21). Retrieved from http://chronicle.com/blognetwork / tenuredradical/2013/07/f-is-for-failure-ordont-invest-your-pension-in-moocs-yet/

Salmon, G. ( 2013). E-tivities: The key to active online learning. Routledge

Siemens, G., and Kathleen M. (2012)."Systemic changes in higher education." in education, 16.1

Strauss, A. L., \& Corbin, J. M. (2015). Basics of qualitative research: Technologues and procedures for Grounded Theory, translated by Ebrahim Afshar, Tehran: Nashr ney(in Persian).

Strauss, A.L, \& Corbin, J.M. (2008). Basics of qualitative research. Translated by Mohammadi, Biok, Tehran: Human Science \& Cultural Studies Center Publication (in Persian).

Ulrich, C., \& Nedelcu, A. (2015). MOOCs in Our University: Hopes and Worries. ProcediaSocial and Behavioral Sciences, 180, 1541-1547. http://doi.org/10.1016/j.sbspro.2015.02.304

Xiao, F., Pardamean, B. (2016)._MOOC Model: Dimensions and Model Design to Develop Learning, The New Educational Review, 43(1): 28-40. 